\title{
Real-time inversion of tsunamis generated by landslides
}

\author{
C. Cecioni ${ }^{1}$, A. Romano ${ }^{1}$, G. Bellotti ${ }^{1}$, M. Risio ${ }^{2}$, and P. de Girolamo ${ }^{2}$ \\ ${ }^{1}$ University of Rome Tre, DSIC, Via Vito Volterra 62, 00161 Rome, Italy \\ ${ }^{2}$ University of L'Aquila, DISAT-LIAM, Via G. Gronchi, 18, 67100 L'Aquila, Italy
}

Received: 20 April 2011 - Revised: 6 July 2011 - Accepted: 12 July 2011 - Published: 22 September 2011

\begin{abstract}
In this paper, we test a method for forecasting in real-time the properties of offshore propagating tsunami waves generated by landslides, with the aim of supporting tsunami early warning systems. The method uses an inversion procedure, that takes input data measurements of water surface elevation at a point close to the tsunamigenic source. The measurements are used to correct the results of precomputed numerical simulations, reproducing the wave field induced by different landslide scenarios. The accuracy of the method is evaluated using the results of laboratory experiments, aimed at studying tsunamis generated by landslides sliding along the flank of a circular shoreline island. The paper investigates what the optimal position is of where to measure the tsunamis, what the effects are, the accuracy of the results, and of uncertainties on the landslide scenarios. Finally, the method is successfully tested using partial input time series, simulating the behaviour of the system in realtime during the tsunami event when forecasts are updated, as the measurements become available.
\end{abstract}

\section{Introduction}

Tsunami inversion techniques are mainly used to reconstruct the properties of the tsunamigenic sources (i.e., coseismic displacement field, slip distribution along the seismic source, etc.) from tsunami records. Among the many previous studies, it is worth to cite Satake (1987), Johnson et al. (1996), Tinti et al. (1996). Most of these researches are based on the numerical and analytical solutions of the linear shallow water equations that are used to compute the propagation of the tsunami waves. To solve the inverse problem (i.e., assessing information on tsunamigenic source from tide-gauges

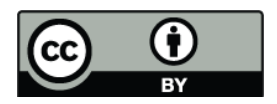

Correspondence to: C. Cecioni (ccecioni@uniroma3.it) records), different approaches, based on Green's function, have been used.

Recently some successful attempts of using the inversion techniques to support real-time tsunami early warning systems have been carried out. Wei et al. (2003) show a method in determining the tsunami waveforms away from the generation area by processing real-time water level records near the tsunamigenic source. Their method extends the previous works by using a long wave model to create a database of synthetic mareograms at a number of strategic locations. Titov et al. (2005) describe the tsunami forecast system adopted by Pacific Marine Environmental Laboratory. This system combines real time seismic and water level data with a forecast database of pre-computed scenarios. The method uses a set of unit sources for constructing a tsunami scenario. Both the inversion methods described the earlier use of Green's function approach.

A different method for tsunami inversion has been proposed by Bellotti et al. (2008). They use a numerical model based on the linearized mild-slope equation, solved in the frequency domain, able to reproduce the propagation of small amplitude tsunami waves. By using one possible scenario of the tsunamigenic source, the model solves the governing equations by providing the Fourier Transform of the free surface elevation. The inversion technique is applied when the surface elevation time series is recorded at some points of the computational domain. The comparison between the numerical pre-computed solution and the Fourier Transform of the measured free surface elevation makes it possible to find, in real-time, a correction parameter in the complex plane, to be applied to each frequency component. Therefore, the method allows for the computing of the tsunami waveform at each point of the computational domain. The procedure takes advantage of the fact that the model equations are linearized. Moreover, the authors have investigated the influence of the length of the input free surface elevation time series using two-dimensional experimental data. By means

Published by Copernicus Publications on behalf of the European Geosciences Union. 
of the analysis of results obtained using partial input time series, they show that the tsunami waveform forecasting is reliable when the first crest has been recorded, and that as the length of the available record increases, the results converge smoothly to the final one.

In this paper we investigate how the model developed by Bellotti et al. (2008), with further improvements by Cecioni and Bellotti (2010a, b), can be applied to support in real-time a landslide-tsunami early warning system, aimed at protecting coasts far away from a potential landslide tsunamigenic source, i.e., the flank of an island, not focussing on the coast of the island itself. The idea of a landslide-tsunami warning system is that devices for the measurement of landslide generated tsunamis are placed close to the possible tsunamigenic area. When a landslide event occurs, records of water surface level are processed in real-time to forecast, at some offshore location, the features of the tsunami, in order to decide if the tsunami alarm has to be spread or not. To test the applicability of the model and to measure the accuracy of the procedure, we use, as reference data, the experimental results of Di Risio et al. (2009), who reproduced tsunamis generated by landslides at the flank of a circular island.

This paper is structured as follows. The next two sections give a brief description of the numerical and the physical models, respectively. Then the results of the inversion procedure applied on the experimental data are shown, along with an investigation on where it is better to locate the sea level recorder device, and an evaluation of the effects of uncertainties on the landslide scenario. Discussion of the results and conclusions close the paper.

\section{Description of the numerical model}

The numerical model is that proposed by Bellotti et al. (2008), which solves the linearized mild-slope equation (MSE hereinafter). We refer to the paper of Bellotti et al. (2008) and Cecioni and Bellotti (2010b) for a detailed description of the equations and only a brief outline of the model is given herein. The MSE (Berkhoff, 1972) describes the small amplitude transient wave propagation on slowly varying depth. In time domain and in terms of free surface elevation $\eta(x, y, t)$, the MSE has the following hyperbolic form:

$$
-\eta_{t t}+\nabla \cdot\left(c c_{\mathrm{g}} \nabla \eta\right)-\left(\omega^{2}-k^{2} c_{\mathrm{g}} c\right) \eta=-\frac{1}{\cosh \left(k h_{\mathrm{b}}\right)} h_{t t}
$$

where $c$ and $c_{\mathrm{g}}$ are the phase and group celerities, respectively, $k$ is the wave number and $\omega$ is the angular frequency; the subscripts indicate derivatives. Cecioni and Bellotti (2010a, b), following Tinti et al. (2006) and Kervella et al. (2007), proposed the introduction of the source term in the right-hand side of Eq. (1), in order to incorporate the wave generation due to sea floor displacements. In Eq. (1), $h(x, y, t)=h_{\mathrm{b}}(x, y)-h_{1}(x, y, t)$ is the water depth equal to the difference between the fixed bottom depth $h_{\mathrm{b}}(x, y)$ and the landslide (or seismic sea floor) elevation $h_{1}(x, y, t)$; its second time derivative is not zero in the area where the landslide (or the earthquake) occurs. The term $1 / \cosh \left(k h_{\mathrm{b}}\right)$ in Eq. (1) represents a filter function, which models the transfer of the bottom movement to the free surface.

It has to be noted that $c, c_{\mathrm{g}}, k$ and $\omega$ in Eq. (1) have been traditionally computed with reference to a single wave frequency component. Therefore, the time domain MSE can be solved by assuming a dominant frequency of the wave spectrum, and the result is valid only for narrow frequency spectra seas. In order to reproduce the frequency dispersion of broad banded spectra, as those of tsunamis, Bellotti et al. (2008), Cecioni and Bellotti (2010a, b) proposed to solve the MSE in the frequency domain. By taking the Fourier Transform of Eq. (1) with respect to the time, it follows

$\nabla \cdot\left(c c_{\mathrm{g}} \nabla N\right)+\omega^{2} \frac{c_{\mathrm{g}}}{c} N=-\frac{1}{\cosh \left(k h_{\mathrm{b}}\right)} \overline{h_{t t}}$.

The resulting elliptic Eq. (2) describes the stationary wave field in terms of $N(x, y, \omega)$, which is the Fourier Transform of the free surface elevation, i.e., relative to a monochromatic wave of single angular frequency $\omega$. The forcing term in the right hand side of Eq. (2) contains $\overline{h_{t t}}$, the Fourier Transform of $h_{t t}$. The model solves a set of equations as Eq. (2), one for each frequency $\omega$, with the appropriate boundary conditions and the values of $c, c_{\mathrm{g}}, k$. The free surface elevation in the time domain, $\eta(x, y, t)$, can be then calculated by taking the Inverse Fourier Transform of the superposition of all the monochromatic solutions $N(x, y, \omega)$.

As already shown in the paper of Cecioni and Bellotti (2010b), when the tsunami generation mechanism is known and its effects on the water are reproduced by the forcing term, the model provides accurate reconstruction of the tsunami scenario. When the model works in a tsunami early warning application, as preliminarily shown in Bellotti et al. (2008), it also makes use of the recording of the tsunami itself, and has to forecast the propagating waveform in realtime while the event is occurring.

A tsunami early warning application of the present model is possible if the computational procedure is split in two parts: one computationally expensive, which has to be carried out before the occurrence of the tsunami; the other, much faster, which provides the forecasting tsunami in real-time using the recording of the tsunami itself. The pre-event computations generate a database of possible tsunami scenario, which are computed assuming a probable generating source, based on the identification of the area where the co-seismic or landslide events likely will occur. The solution of the preliminary computation is the free water surface elevation $\eta^{\mathrm{p}-\mathrm{c}}(x, y, t)$ (where $\mathrm{p}-\mathrm{c}$ stands for pre-computed) and can be stored at any point of the numerical domain.

The second part of the model application starts in realtime when a tsunami occurs and its waveform $\eta^{m}\left(x_{\mathrm{P}}, y_{\mathrm{P}}, t\right)$ 


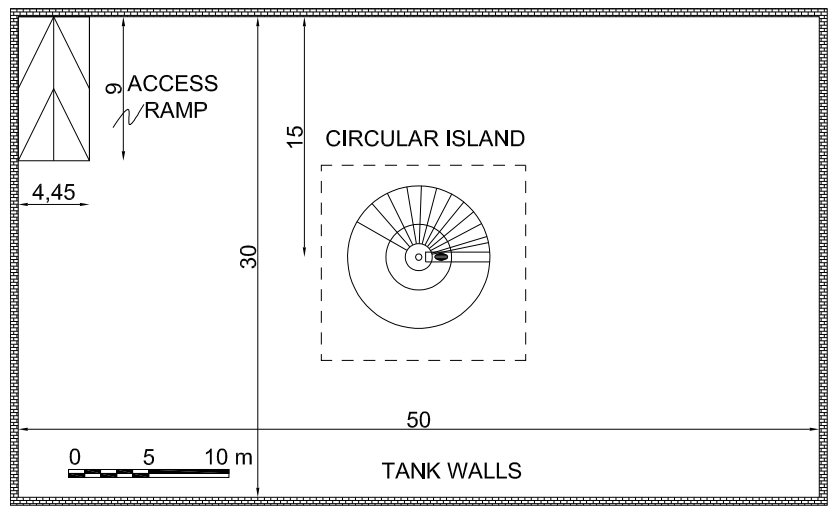

Fig. 1. Sketch of the plan view layout of the laboratory experiments (measures in $\mathrm{m}$ ).

is detected from the sea surface measurement at one position $\left(x_{\mathrm{P}}, y_{\mathrm{P}}\right)$. At that point, we can impose the following identity

$\eta^{m}\left(x_{\mathrm{P}}, y_{\mathrm{P}}, t\right)=s(t) \cdot \eta^{p-c}\left(x_{\mathrm{P}}, y_{\mathrm{P}}, t\right)$

in order to calculate the time varying correction term $s(t)$. Once the correction term is applied to the pre-computed time series, the model solution is equal to the measured one. Indeed, when $s(t)$ is obtained from Eq. (3), it is possible to adjust the pre-event simulation results at any position of the computational domain where no records are available, by using an identical correction term for all the points of the domain:

$\eta(x, y, t)=s(t) \cdot \eta^{\mathrm{p}-\mathrm{c}}(x, y, t)$.

Actually, it is more convenient to apply Eqs. (3) and (4) in the frequency domain. This requires a Fourier Transform of the measured tsunami time series record, $N^{m}\left(x_{\mathrm{P}}, y_{\mathrm{P}}, \omega\right)$. The correction term can, therefore, be defined as follows:

$S(\omega)=\frac{N^{m}\left(x_{\mathrm{P}}, y_{\mathrm{P}}, \omega\right)}{N^{\mathrm{p}-\mathrm{c}}\left(x_{\mathrm{P}}, y_{\mathrm{P}}, \omega\right)}$

and the forecasted tsunami waveform at any point is computed in the frequency domain as:

$N(x, y, \omega)=S(\omega) \cdot N^{\mathrm{p}-\mathrm{c}}(x, y, \omega)$.

As stated, the free surface elevation in the time domain is obtained by means of the Inverse Fourier Transform of $N(x, y, \omega)$. The computation of $S(\omega)$ is carried out in the frequency domain. It has complex values and, therefore, has also the effect of correcting both the amplitude and the phase of each component of the wave spectrum. When switching to the time domain, it is not necessary to synchronize the computed and the measured time series.

An important feature of the model is that the tsunami waveform can be estimated while the tsunami is occurring, in the sense that the term $S(\omega)$ can be calculated even when sea surface elevation record does not contain the entire tsunami

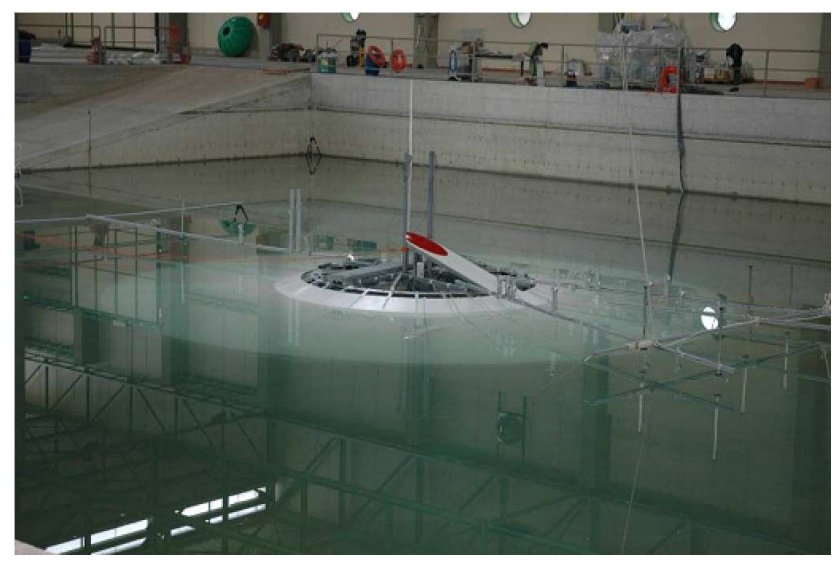

Fig. 2. Picture of the landslide and island models.

waveform. It results that the accuracy of the forecasting model improves with the length of the available registration of the tsunami. However, as we will show later, good estimation of the tsunami waveform can be extracted from the model even when just the first tsunami wave has been recorded.

\section{Description of the physical model}

In order to test the tsunami inversion technique described in the previous section, experimental results have been used. The laboratory experiments (see Di Risio et al., 2009) were carried out in a large wave tank $(50 \mathrm{~m}$ long, $30 \mathrm{~m}$ wide, $3 \mathrm{~m}$ deep) at the Research and Experimentation Laboratory for Coastal Defense (LIC) of the Technical University of Bari, Italy, in cooperation with the Environmental and Maritime Hydraulics Laboratory Umberto Messina (LIAM) of the University of L'Aquila, Italy. The physical model consists of a truncated conical island made up of PVC sheets (thickness $0.01 \mathrm{~m}$ ) and sustained by a steel frame, placed at the centre of the tank. The radius, at the tank bottom level, is $4.45 \mathrm{~m}$. The slope of the flanks of the island is 1:3 (1 vertical, 3 horizontal). The island approximately represents at the Froude scale of 1:1000 the Stromboli island, south Tyrrhenian Sea, Italy (Tinti et al., 2005; Bellotti et al., 2009). Figures 1 and 2 show the plan view of the experimental layout and a picture of the conical island, respectively.

The experiments simulate a landslide body, gravity driven, sliding down over the flank of the conical island. The landslide model is a rigid body, with the shape of a half of the ellipsoid described by the equation $x^{2} / a^{2}+y^{2} / b^{2}+z^{2} / c^{2}=1$, where $a=0.2 \mathrm{~m}, b=0.4 \mathrm{~m}$ and $c=0.05 \mathrm{~m}$, for a total volume $V=0.0084 \mathrm{~m}^{3}$. Di Risio et al. (2009) have tested, in the experimental campaign, different landslide release distances and water depths. The release distance $\zeta$ is defined as the distance between the lower point of the landslide and the undisturbed shoreline measured along the inclined plane. In 


\section{SIDE VIEW}

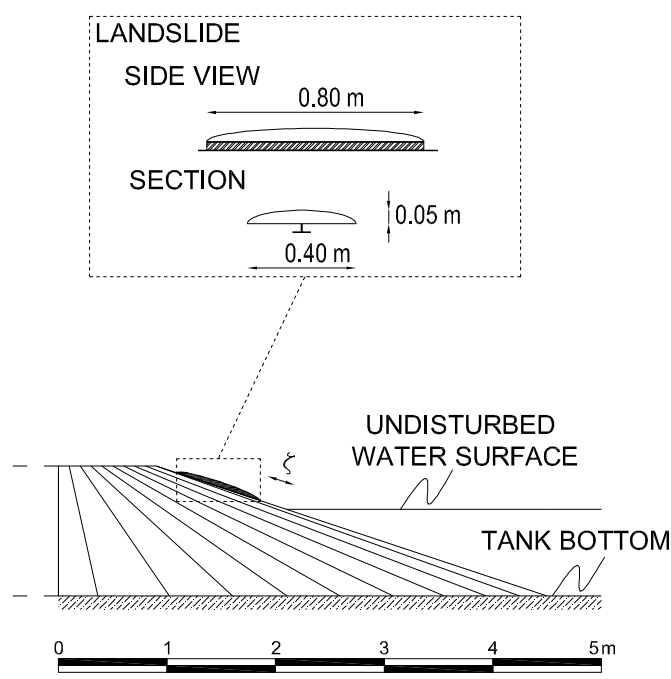

Fig. 3. Layout of the laboratory gauges' positions.

Table 1. Angular and radial position of sea level gauges: the point where the landslide impacts the water is taken as the origin, and the angular position is taken counterclockwise from the landslide motion direction (see Fig. 3).

\begin{tabular}{ccc}
\hline $\begin{array}{c}\text { Gauge } \\
\text { name }\end{array}$ & $\begin{array}{c}\text { Angular position } \\
\theta\left({ }^{\circ}\right)\end{array}$ & $\begin{array}{c}\text { Radial position } \\
r(\mathrm{~m})\end{array}$ \\
\hline $12 \mathrm{~S}$ & 54.3 & 0.63 \\
$20 \mathrm{~S}$ & 44.9 & 0.92 \\
$7 \mathrm{~S}$ & 29.2 & 1.82 \\
$15 \mathrm{~S}$ & -0.3 & 2.37 \\
$24 \mathrm{~S}$ & 0.8 & 4.55 \\
\hline
\end{tabular}

order to test the tsunami inversion technique, we refer to the experimental results for $\zeta=0.30 \mathrm{~m}$ and offshore water depth $h_{\mathrm{b}}=0.80 \mathrm{~m}$.

Traditional resistive gauges were employed to register the instantaneous vertical displacement of the free surface. All the signals have been acquired simultaneously at a frequency of $1000 \mathrm{~Hz}$. The relative position of all the gauges can be found in Fig. 3 and in Table 1. The position of the gauges in Table 1 are expressed in polar coordinates, with the origin at the landslide-water impact point (see Fig. 3) and the angular position measured counterclockwise from the landslide motion direction.

\section{PLAN VIEW}

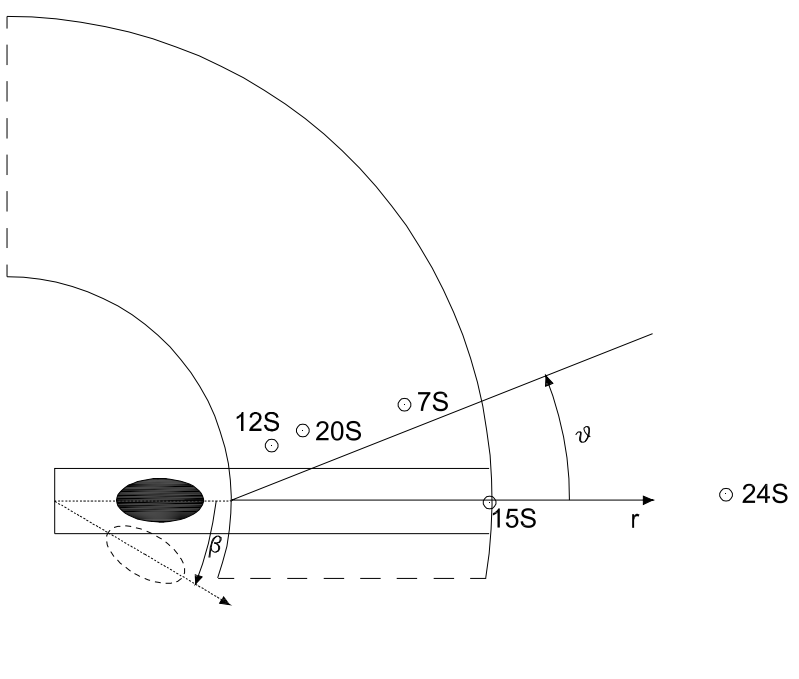

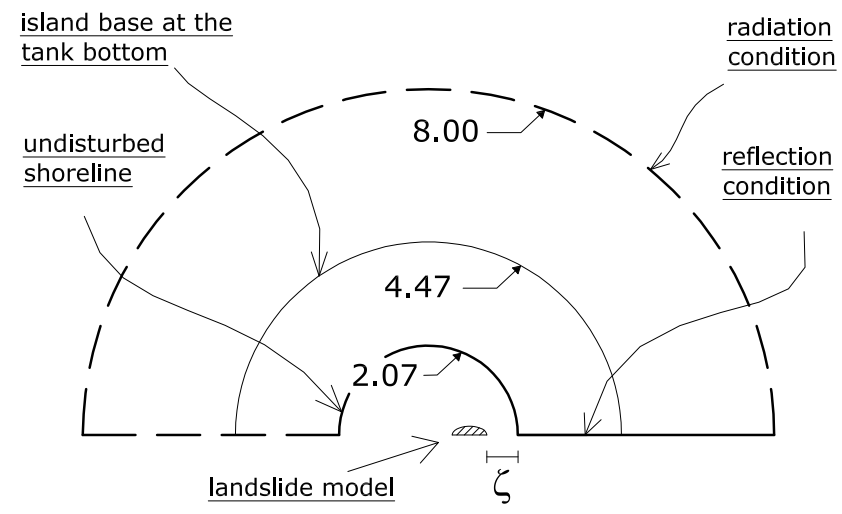

Fig. 4. Sketch of the numerical domain from Cecioni and Bellotti (2010b). The numbers 2.07, 4.47 and 8.00 express the radii in metres of the undisturbed shoreline, the island base at the tank bottom, and the external circular boundary, respectively.

\section{Real-time tsunami inversion}

\subsection{Numerical simulations}

The numerical simulations, presented herein, are aimed at reproducing the conical island experiment. The computational domain is semicircular, as shown in Fig. 4. Taking advantage of the symmetry of the problem across the landslide motion direction, only half of the conical island has been reproduced. The computational domain is limited at the offshore side by a semicircular boundary placed at a distance of $8 \mathrm{~m}$ from the centre of the island.

As sketched in the Fig. 4, at the offshore boundary a radiation boundary condition is applied; it allows the free exit 

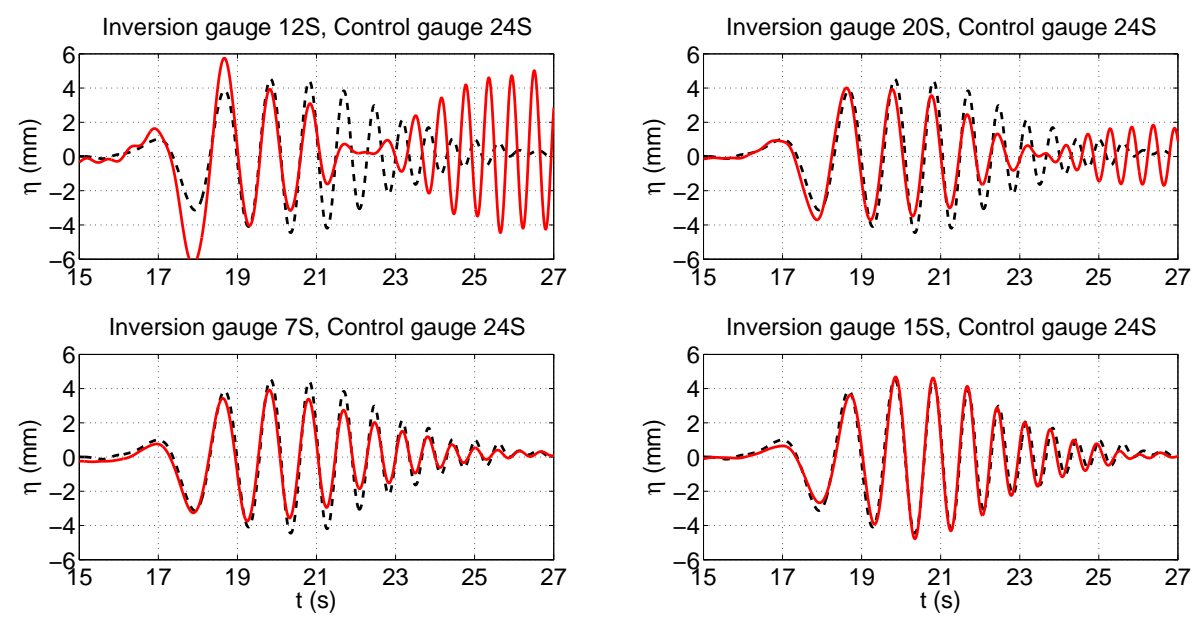

Fig. 5. Comparison of the numerical result (red solid line) with the experimental time series (black dashed line) at the control gauge $24 \mathrm{~S}$. Each plot refers to a different gauge (12S, 20S, 7S and 15S) used for the inversion.

of the propagating waves. A perfect reflection condition is imposed at the undisturbed shoreline, representing a vertical impermeable wall.

The model solves the MSE (2) with the boundary conditions, using a Finite Element Method procedure. The time series reproduced by the model is $50 \mathrm{~s}$ long, with a $\Delta t$ of $0.01 \mathrm{~s}$, and a total of 5000 time steps. In the frequency domain, Eq. (2) has been solved for those long wave components for which relevant energy contents exist. More specifically, 100 discrete angular frequency ranging from $2 \pi \cdot 0.02$ up to $2 \pi \cdot 2 \mathrm{rads}^{-1}$ have been considered for the numerical solution.

The maximum element size of the triangular mesh elements is set in order to ensure at least 10 points for the shortest wave length reproduced $(0.05 \mathrm{~m}$ for all the performed simulations). It results in about 160000 mesh elements and about 81000 Degrees of Freedom of the problem. The computation time for the solution of the 100 discrete angular frequencies is about $12 \mathrm{~min}$ on an AMD Opteron $2462 \mathrm{GHz}$ Computer equipped with 4 GB of RAM.

\subsection{Inversion using the correct landslide scenario}

Firstly, we present results of the inversion procedure applied to the pre-event computations carried out using the correct landslide scenario. This means that the available information on the landslide volume and kinematic from the laboratory experiments are used in the numerical simulations in order to get the forcing term of the MSE (2). In order to estimate the complex correction term $S(\omega)$ of Eq. (5), the numerical model results and one of the collected time series were used. We have, therefore, used the experimental records at one gauge, referred to as "inversion" gauge in the followings, as if it would represent the tsunami record given as input for the inversion procedure. Experimental time series at another gauge located far from the landslide area, referred to as "control gauge" in the following, is used to validate the model prediction of the propagating waves. The key point here is to analyse where it would be better to locate the inversion sea level gauge, which will record the tsunami and will provide in real-time the input for the inversion procedure. On one hand, in order to give early predictions, it is desirable to use gauges very close to the sub-aerial landslide; on the other hand, the model is expected to perform better in deep water, so in order to get accurate results it is desirable to use gauges located not in the very near field.

In order to investigate what position of the inversion gauge will provide better accuracy of the tsunami prediction, Fig. 5 reports the results of 4 different inversions, obtained using as input time series those of the gauges $12 \mathrm{~S}(r=0.63 \mathrm{~m}$, $\left.\theta=54.3^{\circ}\right), 20 \mathrm{~S}\left(r=0.92 \mathrm{~m}, \theta=44.9^{\circ}\right), 7 \mathrm{~S}(r=1.82 \mathrm{~m}$, $\left.\theta=29.2^{\circ}\right)$ and $15 \mathrm{~S}\left(r=2.37 \mathrm{~m}, \theta=-0.3^{\circ}\right)$. The numerical results (red solid line) are checked at the control gauge $24 \mathrm{~S}$ $\left(r=4.55 \mathrm{~m}, \theta=0.8^{\circ}\right)$ by comparison with the experimental records (black dashed line). It appears that the performances of the inversion procedure improve as the distance of the inversion gauge from the source increases. This is clearly related to the fact that in the very near field the model results are not satisfactory, see, for example, the results reported on Fig. 5 of Cecioni and Bellotti (2010b). As shown in that paper, the accuracy of the results deteriorates in the very near field. This is mostly related to the fact that the present model does not reproduce complicated three-dimensional flows and nonlinear effects; both are expected to play a role close to the landslide and in shallow waters. Results obtained using as inversion gauge, the $12 \mathrm{~S}$ are of very poor quality. When the inversion gauge $20 \mathrm{~S}$ is used, the results are good for the largest waves, but the following smaller waves are not correctly reproduced. The computations obtained using the gauge $7 \mathrm{~S}$ give a good reproduction of the largest waves and 

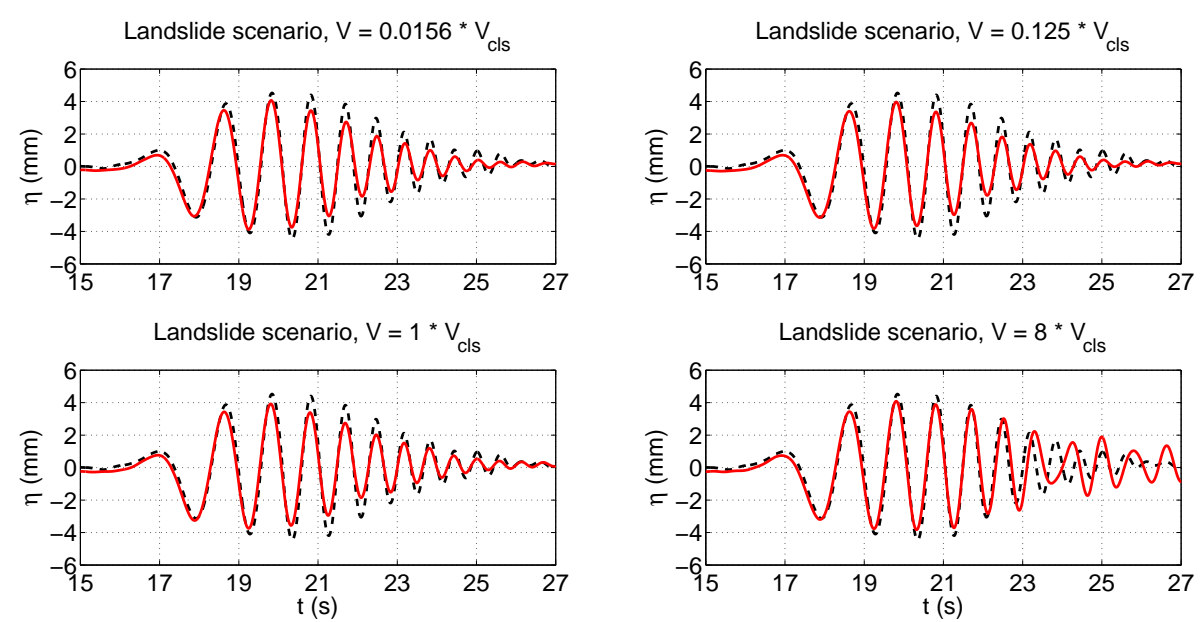

Fig. 6. Comparison of the numerical result (red solid line) with the experimental registration (black dashed line) at the control gauge $24 \mathrm{~S}$. Numerical model prediction is performed adopting gauge $7 \mathrm{~S}$ as the inversion gauge, and using pre-event numerical computations carried out with 4 tentative landslide volumes. $V_{\mathrm{cls}}$ is the volume of the so called correct landlide scenario.
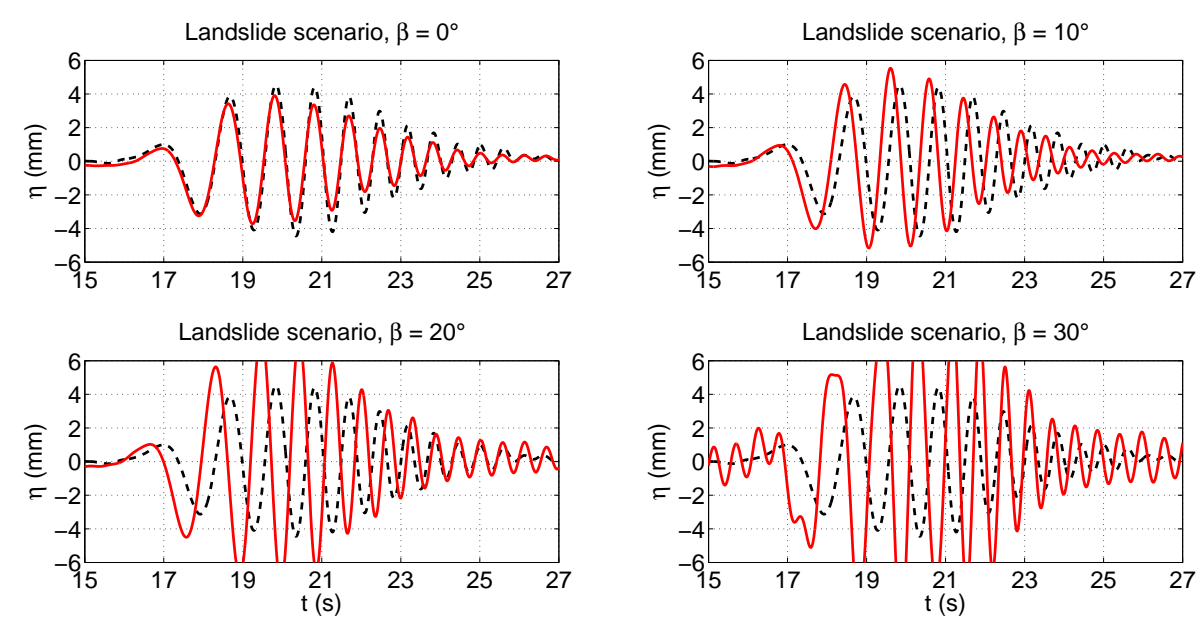

Fig. 7. Comparison of the numerical result (red solid line) with the experimental records (black dashed line) at the control gauge $24 \mathrm{~S}$, using inversion gauge 7S. Numerical model prediction is performed using pre-event numerical computations carried out with 4 tentative landslide motion directions. The angle $\beta$ measures the difference between the correct landslide scenario and that used in the computations. $\beta=0^{\circ}$ represents the correct landslide scenario, i.e., that representing the experiments.

of the trailing wave train. Very accurate results are obtained using the very far gauge $15 \mathrm{~S}$. Note, however, that the instrument is on the landslide path and in the field it is not feasible to place wave gauge at that position, since they would be certainly damaged by landslides. In the following, the gauge $7 \mathrm{~S}$ is taken as the inversion one, while the gauge $24 \mathrm{~S}$ is used as the control one, representative of waves radiating offshore.

\subsection{Effect of uncertainties on the landslide scenario}

It is now evaluated the impact on the accuracy of the results of using pre-event computations carried out with wrong landslide scenarios. Of course it is impossible to know exactly the properties of the landslide and its kinematic before the tsunami event and it is, therefore, very important to assess the effects of scenario uncertainties on the method presented. The effect of uncertainty of three parameters is separately evaluated here: the volume of the landslide, its position (i.e., the axis along which it slides), and its kinematic (i.e., its velocity). Other sources of uncertainty, such as the shape of the landslide and the porosity, are not considered herein.

Figure 6 presents the results at the control gauge 24S, obtained applying the inversion from the sea level record at gauge 7S, using 4 landslide scenarios, including the correct one. These have been computed by using landslides of different dimensions, but keeping the same ellipsoidal shape. The 4 landslides have been obtained by scaling the original one 

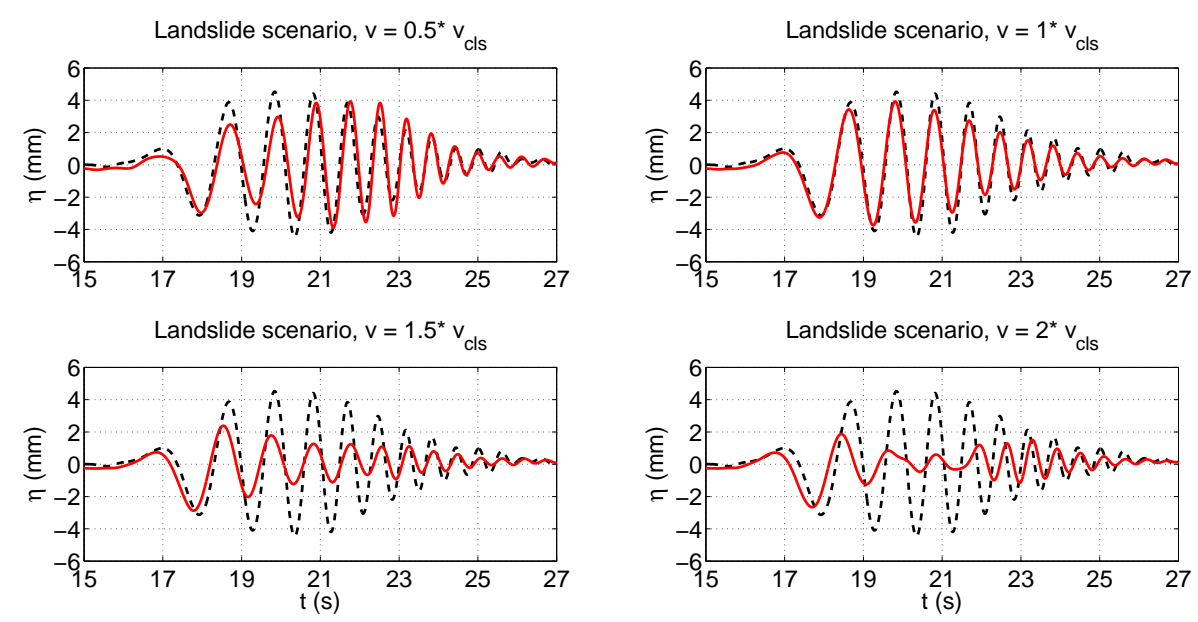

Fig. 8. Tsunami inversion using scenario computations with wrong landslide velocity; inversion from gauge $7 \mathrm{~S}$, results checked against records at gauge $24 \mathrm{~S}$. Red solid lines refer to numerical results, black dashed lines to experimental time series. $v_{\text {cls }}$ is the velocity of the so called correct landslide scenario.

used in the physical model, multiplying the axes by 0.25 , $0.50,1.00$ and 2.00. The resulting landslide volumes measure $0.0156,0.1250,1.0000$ and 8.0000 , respectively, times the volume of the reference landslide (i.e., the one reproduced in the physical model). Again the red line reports the numerical model prediction, while the black dashed line refers to the experimental data.

The results indicate that the inversion procedure is not very affected by the uncertainty on landslide volume. As far as the largest waves are concerned, no differences can be seen, nor a trend with the volume of the landslide. It is, however, pointed out that the train of small waves that follows the largest, are better reproduced by the small and the correct landslide scenarios, while increasing the dimension of the landslide a poor accuracy is obtained. Of course, as the method is aimed at predicting the largest waves, it can be concluded that the effect of uncertainties on the landslide volume can be neglected.

Let us now analyse the effect of uncertainties on the landslide position, i.e., on the direction of the landslide motion on the flank of the island. The reference position is that used in the experiments (see Fig. 3). The pre-event numerical computations are created assuming landslide scenarios with the sliding axis rotated on an angle $\beta$ equal to 10,20 and 30 degrees from the referenced one. Figure 7 presents the sea level oscillations at the control gauge $24 \mathrm{~S}$ given by the numerical model, obtained using the inversion gauge $7 \mathrm{~S}$ and the 4 preevent computations with different landslide motion direction. Comparing the numerical results (red line) with the physical model records (black dashed line) it can be noted that the position of the landslide plays a very important role on the procedure. The accuracy of the results given by the method decreases as the direction of the sliding axis diverges from the correct one. An error of $10^{\circ}$ on the landslide position gives already poor results; an error of $30^{\circ}$ appears to produce unacceptable predictions.

Finally, the effect of uncertainties on the landslide velocity is investigated. For simplicity the pre-event computations are carried out using the same kinematic law derived from the laboratory experiment, but velocities are multiplied by $0.5,1.0,1.5$ and 2.0 , respectively. Thus reproducing one slower and two faster falls than the referenced one. The results of the inversion procedure, again referred to the control gauge $24 \mathrm{~S}$ and obtained from the inversion gauge $7 \mathrm{~S}$, are reported in Fig. 8. The comparison with the laboratory records (black dashed line) indicates that an underestimation of the landslide velocity in the pre-event simulation still produces acceptable results after the inversion procedure. On the contrary, the fast landslide scenarios give poor accuracy results, and the forecasted wave packet is not able to reproduce the largest surface elevation recorded at the control gauge.

It can be concluded that the pre-event uncertainties on the landslide volume seems not to affect the inversion forecasting results, while the landslide falling position appears to have a strong influence upon the accuracy of the method. Also very small errors on the position of the axis along which the landslide moves, induce large errors on the results. Furthermore, it appears that it is better to reproduce slower landslides, rather than selecting too fast landslide scenarios.

\subsection{Real-time inversion}

The ability of the procedure to deal with truncated input time series is now evaluated. Of course, when performing inversion in real-time, the data are used as they become available. Results of an example computation performed with the correct scenario are reported in Fig. 9. Left panels refer to the time series at the inversion gauge $7 \mathrm{~S}$; the black dashed line refers to the full record, identical for all the plots, and the 

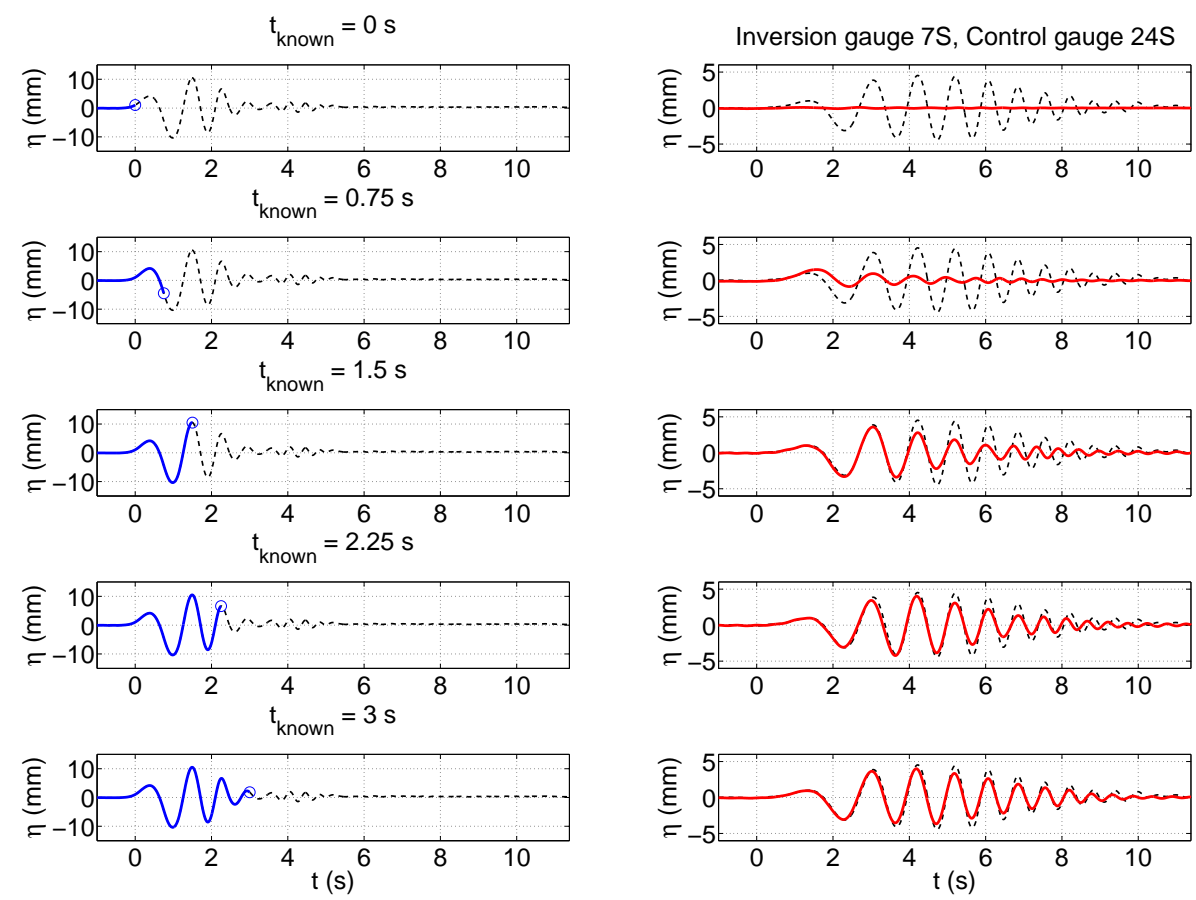

Fig. 9. Sample computations of real-time inversion using partial input time series at gauge 7S; results checked against records at gauge $24 \mathrm{~S}$.

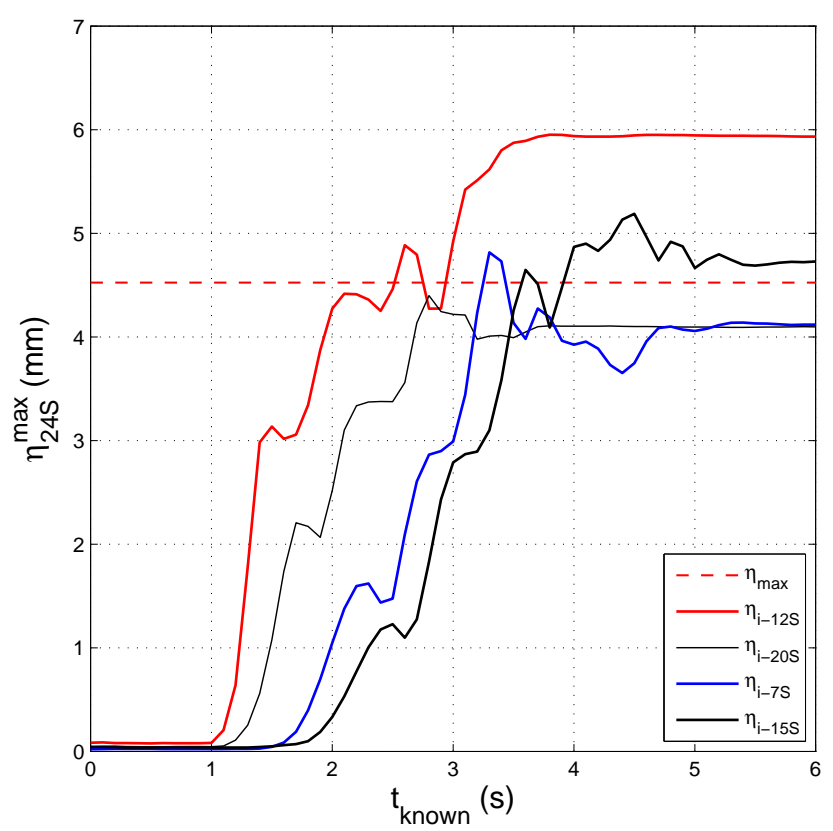

Fig. 10. Predicted maximum surface elevation at gauge $24 \mathrm{~S}$, using as input partial time series of length $t_{\text {known }}$; horizontal dashed line represents the reference experimental result at gauge $24 \mathrm{~S}$.

blue solid one to the part of the signal considered available for each plot. Right panels show the experimental time series at the control gauge $24 \mathrm{~S}$ (black dashed lines) and those predicted by the model (red solid line). Each row of plots refers to a specific elapsed time, measured by the parameter $t_{\text {known }}$, of the input time series used for the inversion. For instance, the first row considers an input time series available up to $0 \mathrm{~s}$, selected as origin of the time: no waves are measured and, therefore, no waves are predicted at gauge $24 \mathrm{~S}$. The second row refers to the computation carried out after $0.75 \mathrm{~s}$, after the first wave crest has been measured: the predicted time series shows an underestimated wave train at gauge $24 \mathrm{~S}$. When the first wave has been completely measured, and the crest of the second wave (the largest) is included in the input time series, the predictions appear to be quite close to the reference experimental data, as shown by the plots of the third row. As the input time series becomes longer, the results converge to the measured ones. From Fig. (9) it appears that the leading wave is well predicted already at $t_{\text {known }}=1.5 \mathrm{~s}$, while the following wave packet is still underestimated.

The convergence process is also shown in Fig. 10, where the effect of changing the inversion gauge is also evaluated. Here the maximum surface elevation predicted by the procedure at the control gauge $24 \mathrm{~S}$, is plotted against the length of the input time series. The 4 lines refer to results obtained using as input time series those of gauges $12 \mathrm{~S}, 20 \mathrm{~S}, 7 \mathrm{~S}$ and $15 \mathrm{~S}$. The horizontal dashed line represents the maximum experimental surface elevation at the control gauge $24 \mathrm{~S}$, i.e., the correct final results. It appears that the convergence procedure is relatively smooth. Applying the inversion procedure using very near-source gauges (12S and 20S) provides a faster, but less accurate estimation of the maximum sea surface elevation. The inversion carried out using the very far gauge is more accurate, but takes longer to give the final results. 


\section{Discussion and conclusions}

The inversion procedure presented in this paper seems to provide accurate forecasts of the tsunamis and is able to work in real time, using partial input time series. It seems, therefore, that it can be a useful tool to support tsunami early warning systems.

One potential drawback of the procedure is that the accuracy of the results deteriorates if the pre-computed landslide scenario used for the inversion is not adequate. Among the several sources of uncertainties about the scenario, the results show that the landslide dimension and the kinematic do not play a relevant role. On the contrary, the position of the axis along which the landslide falls, appears to have a large effect.

A possible method for reducing the effects of scenario uncertainties is that of trying to select the most appropriate one using the measurements in real-time. The simplest approach is that of using two measurement devices of the free surface elevation. One input time series can be used to forecast, in the position of the second device, the time history of the surface elevation for each of the pre-computed scenarios. Then, by comparing the results and the measurements at the second device, it may be possible to decide what is the scenario that better describes the event.

However, in the practice, monitoring systems of unstable flanks may be used to obtain reasonable estimates of the position, shape and dimension of potential subaerial landslides. This information may be used to prepare specific landslide scenarios to be included in the database. An example of such a measurement technique is shown in Casagli et al. (2010), who have set-up a ground-based radar interferometry system to monitor the Sciara Del Fuoco unstable flank at Stromboli.

Measurements of tsunamis, essential in the present technique, can be obtained using pressure transducers. Nevertheless, it has to be stressed that waves generated by landslides are not very long, and a "large portion of the wave packet energy pertains to deep water waves" (Bellotti et al., 2009). Then pressure transducers placed at the sea bottom may not be able to properly measure these tsunamis. A possible alternative is to mount the pressure transducers on poles or, for high water depths, on mooring lines of floating devices, so that the level at which measurements are carried out can be selected independently from the water depth. In the latter case, care has to be used to compensate the measurements for the possible movements of the instruments; these are expected to induce further pressure components, not related with surface waves. It is also worth mentioning that the measurements have to be processed in real time, using detection algorithms (Bressan and Tinti, 2011; Beltrami, 2008), since the procedure presented in this paper expects as input purely the tsunami data. In particular, when measurement devices are placed close to the water surface, they can measure also wind-waves components and special algorithms have to be applied (e.g., McGehee and McKinney, 1995; Beltrami and Di Risio, 2011).
The paper has presented a brief analysis of how the position of the inversion gauge influences the accuracy of the procedure. As stated, it is desirable to measure the tsunamis close to the source, in order to increase the amount of time for the spreading of the alert. However, the propagation model used in the procedure performs better in deep waters, so the accuracy of the results can be improved by placing the inversion gauge not too close to possible landslides. In the practice, it is also important to place the instruments on a stable sea bottom area, that is not expected to be reached by the landslide material.

The computations presented in this paper refer to a subaerial landslide only, as the experimental data available so far do not include submerged ones. It is certainly desirable to evaluate the performances of the method for submerged landslides, for which monitoring systems are not able to provide information and warning on flank instabilities. This is one of the points that will be addressed in our ongoing research, since new experimental data will extend the results of Di Risio et al. (2009), including submerged landslides in the next future.

Acknowledgements. This work was carried out under the research project PRIN 2007 ("Development and validation of hydraulic and geologic tools for supporting a Tsunami Early Warning System. Implementation to the Stromboli (Eolie) landslide case.") and FIRB 2008-FUTURO IN RICERCA ("Design, construction and operation of the Submarine Multidisciplinary Observatory experiment"), both funded by the Italian Ministry for University and Scientific Research (MIUR).

Edited by: S. Tinti

Reviewed by: F. Dias and D. Tappin

\section{References}

Bellotti, G., Cecioni, C., and De Girolamo P.: Simulation of small-amplitude frequency-dispersive transient waves by means of the mild-slope equation, Coast. Eng., 55(6), 447-458, doi:10.1016/j.coastaleng.2007.12.006, 2008.

Bellotti, G., Di Risio, M., and De Girolamo, P.: Feasibility of Tsunami Early Warning Systems for small volcanic islands, Nat. Hazards Earth Syst. Sci., 9, 1911-1919, doi:10.5194/nhess-91911-2009, 2009.

Beltrami, G. M.: An ANN algorithm for automatic, real-time tsunami detection in deep-sea level measurements, Ocean Eng., 35(5-6), 572-587, doi:10.1016/j.oceaneng.2007.11.009, 2008.

Beltrami, G. M. and Di Risio, M.: Algorithms for automatic, real-time tsunami detection in wind-wave measurements, Part I: Implementation strategies and basic tests, Coast. Eng., 58(11), 1062-1071, doi:10.1016/j.coastaleng.2011.06.004, 2011.

Berkhoff, J. C. W.: Computation of combined refraction-diffraction, Proceedings of the 13th International Conference On Coastal Engineering-ASCE, Vancouver, Canada, 1972.

Bressan, L. and Tinti, S.: Structure and performance of a realtime algorithm to detect tsunami or tsunami-like alert conditions 
based on sea-level records analysis, Nat. Hazards Earth Syst. Sci., 11, 1499-1521, doi:10.5194/nhess-11-1499-2011, 2011.

Casagli, N., Catani, F., Del Ventisette, C., and Luzi, G.: Monitoring, prediction and early warning using ground-based radar interferometry, Landslides, 7(3), 291-301, doi:10.1007/s10346-0100215-y, 2010.

Cecioni, C. and Bellotti, G.: Modeling tsunamis generated by submerged landslides using depth integrated equations, Appl. Ocean Res., 32, 343-350, doi:10.1016/j.apor.2009.12.002, 2010a.

Cecioni, C. and Bellotti, G.: Inclusion of landslide tsunamis generation into a depth integrated wave model, Nat. Hazards Earth Syst. Sci., 10, 2259-2268, doi:10.5194/nhess-10-2259-2010, 2010.

Di Risio, M., De Girolamo, P., Bellotti, G., Panizzo, A., Aristodemo, F., Molfetta, M. G., and Petrillo, A. F.: Landslidegenerated tsunamis runup at the coast of a conical island: new physical model experiments, J. Geophys. Res., 114, C01009, doi:10.1029/2008JC004858, 2009b.

Johnson, J. M., Satake, K., Holdahl, S. R., and Sauber, J.: The 1964 Prince William Sound earthquake: joint inversion of tsunami and geodetic data, J. Geophys. Res., 101, 523-532, doi:10.1029/95JB02806, 1996.

Kervella, Y., Dutykh, D., and Dias, F.: Comparison between three dimensional linear and nonlinear tsunami generation models, Theor. Comput. Fluid Dynam., 21, 245-269, 2007.

McGehee, D. and McKinney, J.: Tsunami detection and warning capability using nearshore submerged pressure transducers - Case study of the 4 October 1994 Shikotan Tsunami. Proceedings of the 4th International Tsunami Symposium, IUGG, Boulder, Colorado, 133-144, 1995.
Satake, K.: Inversion of tsunami waveforms for the estimation of a fault heterogeneity: Method and numerical experiments, J. Phys. Earth., 35, 241-254, 1987.

Tinti, S., Piatanesi, A., and Bortolucci, E.: The finite-element wave propagator approach and the tsunami inversion problem, J. Phys. Chem. Earth, 21, 27-32, doi:10.1016/S0079-1946(97)00005-0, 1996.

Tinti, S., Manucci, A., Pagnoni, G., Armigliato, A., and Zaniboni, F.: The 30 December 2002 landslide-induced tsunamis in Stromboli: sequence of the events reconstructed from the eyewitness accounts, Nat. Hazards Earth Syst. Sci., 5, 763-775, doi:10.5194/nhess-5-763-2005, 2005.

Tinti, S., Pagnoni, G., and Zaniboni, F.: The landslides and tsunamis of the 30th of December 2002 in Stromboli analysed through numerical simulations, Bullettin of Volcanology, 68(5), 462-479, doi:10.1007/s00445-005-0022-9, 2006.

Titov, V. V., González, F. I., Bernard, E. N., Eble, M. C., Mofjeld, H. O., Newman, J. C., and Venturato, A. J.: Real-time tsunami forecasting: Challenges and solutions, Nat. Hazards, 35(1), 4158, 2005.

Wei, Y., Cheung, K. F., Curtis, G. D., and McCreery, C. S.: Inverse algorithm for tsunami forecasts, J. Waterw. Port Coast. Ocean Eng., 129(3), 60-69, doi:10.1061/(ASCE)0733950X(2003)129:2(60), 2003. 